\title{
High expression of TFEB is associated with aggressive clinical features in colorectal cancer
}

This article was published in the following Dove Press journal:

OncoTargets and Therapy

Jing Liang ${ }^{1, *}$

Xinfeng Jia ${ }^{2, *}$

Kun Wang ${ }^{3, *}$

Niankun Zhao ${ }^{4}$

'Department of Oncology, Tianjin Binhai New Area Dagang Hospital, Tianjin 300270, China; ${ }^{2}$ Geriatrics Department, Tianjin Civil Affairs Bureau Geriatric Hospital, Tianjin 3001 II, China; ${ }^{3}$ Department of Genitourinary Oncology, Tianjin Medical University Cancer Institute and Hospital, National Clinical Research Center for Cancer, Key Laboratory of Cancer Prevention and Therapy, Tianjin, Tianjin's Clinical Research Center for Cancer, Tianjin 300030, China; ${ }^{4}$ Department of Internal Medicine, Tianjin Binhai New Area Dagang Hospital, Tianjin 300270, China

*These authors contributed equally to this work
Correspondence: Jing Liang Department of Oncology, Tianjin Binhai New Area Dagang Hospital, Nanhuan Road I200, Binhai New Area, Tianjin 300270, China

Email liangjing_blue@I63.com
Objectives: The transcription factor EB (TFEB), a member of the micropthalmia family, has been found to be associated with autophagy and upregulated in some kinds of tumors. However, very few studies focused on TFEB in colorectal cancer (CRC). TFEB expression status and its relevance to clinical features in CRC would be analyzed in this study.

Materials and methods: Real-time PCR, Western blot, and immunohistological staining were used to evaluate TFEB expression in CRC tissues and adjacent normal tissues, and the role of TFEB in CRC cell lines was investigated in vitro and in vivo.

Results: TFEB was expressed at lower level in CRC tissues than normal in both mRNA and protein level. However, there were significantly positive correlations between TFEB expression in cancer tissues and malignant progression of CRC. Cancers with TFEB overexpression always had deeper infiltration and higher lymphatic metastasis rate. Furthermore, patients with high TFEB levels always had poor survival, and higher TFEB expression could be a predictor of survival in multivariate analysis. Meanwhile, knockdown TFEB by shRNA or knockout TFEB by sgRNA in CRC cell lines could significantly inhibit cell proliferation and migration in amino acid-free medium. In addition, we found a positive relationship between TFEB and Beclin1 expression, and silencing TFEB inhibited Beclin1 expression in CRC cells.

Conclusion: TFEB expression correlated with aggressive clinical features in CRC, and higher TFEB expression could be a prognostic factor and potential treatment target of CRC.

Keywords: TFEB, colorectal cancer, clinicopathologic variables, prognostic factor, Beclin1

\section{Introduction}

Colorectal cancer (CRC) is a cancer with a high morbidity level and a high lethality rate worldwide. ${ }^{1,2}$ Aggressive CRC is a term commonly used to describe CRC with a poor prognosis, and aggressive clinical features of CRC always mean poor differentiation grade, positive metastasis status, and/or higher clinical Dukes stage. Even with advanced treatment with a combined approach of routine therapy and molecular targeting treatment, the median survival time was not significantly improved. ${ }^{3}$ Still, $>50 \%$ of patients develop metastasis and recurrence after surgery. ${ }^{4,5}$ Therefore, early diagnosis and therapy hold a significant prognostic value. Meanwhile, identifying new biomarkers as well as specific therapeutic targets for CRC would improve the prognosis of CRC patients. ${ }^{6,7}$

Among many factors and mechanisms that are involved in $\mathrm{CRC}$, researchers find that autophagy plays an important role when an enterocyte shifts from normal to malignant cell. ${ }^{8,9}$ Autophagy is a catabolic process of "self-eating" to destroy and recycle the unnecessary or damaged cell components to satisfy the needs of their growth in most cell types; hence, it plays an important role in maintaining cellular homeostasis. ${ }^{10,11}$ The whole process of autophagy is regulated by multiple proteins. ${ }^{12}$ 
Transcription factor EB (TFEB) is a master transcription factor directing the expression of hundreds of autophagy- and lysosomal-related genes. ${ }^{13}$ TFEB could be phosphorylated at nutrient-rich condition and then bound to 14-3-3, which is anchored in the cytoplasm of cells. At the condition of starvation, TFEB is dephosphorylated and translocated to the nucleus, in which it initiates the transcription of target genes involved in autophagy and lysosomal function. ${ }^{14}$ Beclin1, an ortholog of the Atg6 in yeast, plays an important role in a critical step of the autophagic process, by interacting with PI3K, which facilitates lipid membrane extension, cargo recruitment, and autophagosome maturation. ${ }^{12}$ Some studies have found that TFEB could affect the expression of Beclin1 in cancer cells. ${ }^{11,17}$

TFEB has been found to be upregulated in many kinds of tumors; ${ }^{15-17}$ however, very few studies focused on TFEB in CRC. Only one paper has shown that the expression of TFEB in tumors was significantly lower than normal tissues. ${ }^{18}$ In this study, we investigated the expression level of TFEB in $\mathrm{CRC}$ tissues and adjacent normal tissues and analyzed the correlation of TFEB with clinicopathological variables and survival of CRC patients.

\section{Materials and methods}

\section{Patients and tissues}

A total of $120 \mathrm{CRC}$ tissues and adjacent tissues were obtained from patients who underwent radical resection at Tianjin Binhai New Area Dagang Hospital during 2009-2013. Five pairs of fresh tissues were collected from Tianjin Binhai New Area Dagang Hospital in 2017. All the patients were diagnosed by two pathology doctors according to the guideline of the World Health Organization and not treated with neoadjuvant therapy. This study complied with the Declaration of Helsinki and was approved by the Human Ethics and Research Ethics Committees of the hospital. In addition, written informed consents were obtained from all patients.

\section{Antibodies}

The primary antibodies used in this study are listed as follows: TFEB (Abcam, Cambridge, MA, USA), Beclin1 (Proteintech, Chicago, IL, USA), Ki-67 (Santa Cruz Biotechnology Inc., Dallas, TX, USA), caspase3 (Abcam), and GAPDH (Santa Cruz Biotechnology Inc.). The anti-mouse secondary antibodies (Santa Cruz Biotechnology Inc.), and anti-rabbit secondary antibodies (Zhongshan Goldbridge Biotechnology, Beijing, China) were purchased for Western blot. The secondary antibody PV6001 was obtained from Zhongshan Goldbridge Biotechnology for immunohistochemistry (IHC) assay.
RNA isolation and real-time PCR analysis

Total RNA was extracted using TRIzol reagent (Thermo Fisher Scientific, Waltham, MA, USA), and $1 \mu \mathrm{g}$ of total RNA was reverse transcribed to cDNA using a Reverse Transcription Kit (Qiagen NV, Venlo, the Netherlands). Real-time PCR analyses were performed using SYBR-Green quantitative real-time PCR Master Mix Kit (Toyobo Co., Osaka, Japan). The primer sequences used were as follows: TFEB forward: 5'-GGTGCAGTCCTACCTGGAGA-3', reverse: 5'-GTGGGCAGCAAACTTGTTCC-3'; GAPDH forward: 5'-AACGTGTCAGTGGT GGACCTG-3', reverse: 5'-AGTGGGTGTCGCTGTTGAAGT-3'.

\section{Western blot}

Equal amount of proteins was separated by SDS-PAGE and then transferred to polyvinylidene fluoride (PVDF) membrane (EMD Millipore, Billerica, MA, USA); 5\% nonfat dry milk was used to block PVDF membrane for 1 hour. The above PVDF membrane was incubated with the corresponding primary antibodies for overnight and then incubated with HRP-conjugated secondary antibodies for 1 hour at room temperature. Detection was performed by enhanced chemiluminescence (ECL) Western blotting kit.

\section{IHC assay}

Sections were deparaffinized and rehydrated; 3\% hydrogen peroxide was used to block endogenous peroxidase, and then the sections were boiled in $10 \mathrm{mM}$ citrate buffer ( $\mathrm{pH}$ 6.0) for 3 minutes. After that, the sections were incubated with TFEB antibody (1:100 dilution) or Beclin1 (1:100 dilution) overnight at $4^{\circ} \mathrm{C}$. Then the sections were incubated with PV6001 for 1 hour at $37^{\circ} \mathrm{C}$ and stained with DAB for 2 minutes. Five high-power fields from each slice were chosen and scored, the positive cells among 100 cells were counted in a $40 \times 10$ magnification manually. Patients with TFEB expression levels of $\leq 50 \%$ in sections were assigned to the low-expression group, whereas those with values $>50 \%$ were assigned to the high-expression group.

\section{Cell culture}

HCT-116 and LoVo were purchased from American Type Culture Collection (ATCC, Manassas, VA, USA). These cells were maintained in DMEM containing $10 \%$ of FBS, $100 \mathrm{U} / \mathrm{mL}$ of penicillin, and $100 \mu \mathrm{g} / \mathrm{mL}$ of streptomycin (Thermo Fisher Scientific) at $37^{\circ} \mathrm{C}$ with $5 \% \mathrm{CO}_{2}$.

\section{Plasmid construction and cell infection}

shRNAs were constructed into pLKO.1 plasmids. The shKHK sequence was 5'-TGGCAACAGTGCTCCCAATAG-3' and 
was synthesized by Shanghai Genechem Co., Ltd. (Shanghai, China). sgctrl and sgTFEB were designed according to the Zhang laboratory's protocol ${ }^{19}$ and then cloned into the lentiCRISPR/CAS9 vector. The sgTFEB sequence targeted 5'-CATTGACAACATTATGCGTC-3'. For TFEB overexpression, the gene sequences encoding TFEB was amplified from HCT-116 and cloned into the pcDNA3.1 plasmid according to the manufacturer's instructions. Lipofectamine 2000 Reagents (Thermo Fisher Scientific) were used for cell transfection.

\section{Cell Counting Kit-8 (CCK-8) for cell proliferation}

The cells were seeded in a 96-well plate at a density of $1 \times 10^{3}$ cells per well; $10 \mu \mathrm{L} \mathrm{CCK-8}$ was added to the wells at different time points, and then absorbance was measured at $450 \mathrm{~nm}$ by a microplate reader.

\section{Colony formation assay}

A total of $2 \times 10^{3}$ cells/well were seeded in a six-well plate and cultured for 10-14 days. Colonies were fixed with $10 \%$ formaldehyde for 30 minutes and stained with $0.5 \%$ crystal violet for 5 minutes. Then the colonies were photographed and analyzed.

\section{In vitro migration assay}

Cell migration was assessed using a Boyden chamber assay. Cells $\left(5 \times 10^{5}\right)$ suspended in serum-free medium were transferred to the upper chamber $(8 \mu \mathrm{M}$; Costar, Cambridge, MA, USA), and the latter were seeded in complete medium. After 24 hours, cells that had migrated to the bottom side of the membrane were fixed in $70 \%$ ethanol and stained with crystal violet.

\section{Luciferase reporter assay}

A reporter construct containing Beclin1 promoter was linked to a luciferase reporter gene, then CRC cell lines HCT116 and LoVo were cotransfected with $0.5 \mu \mathrm{g}$ pGL3-Beclin1, $0.1 \mu \mathrm{g}$ CMV- $\beta$-gal plasmid, and $1 \mu \mathrm{g}$ shTFEB or shctrl plasmid in six-well plates using Fugene $6^{\circledR}$ reagent (Promega Corporation, Fitchburg, WI, USA). After transfection, cells were lysed 48 hours and assayed for luciferase and $\beta$-galactosidase activities, with the former being normalized by the latter.

\section{In vivo proliferation assay}

Male BALB/c nude mice (4-5 weeks old) were purchased from the Model Animal Research Center of Nanjing University (Nanjing, China). The protocol was approved by the responsible governmental animal ethics committee. A total of $2 \times 10^{6}$ HCT116 cells were inoculated subcutaneously in the right flank of the nude mice. Tumors were measured every 4 days, and tumor volume was calculated by length $\times$ width $\times$ width $/ 2$. Meanwhile, Ki-67 and caspase 3 were also detected by IHC assay to analyze the tumor proliferation in vivo. All animal studies were approved by the Dagang Hospital in accordance with the principles and procedures outlined in the NIH Guide for the Care and Use of Laboratory Animals.

\section{Statistical analysis}

SPSS 19.0 was used. Chi-squared test was used to assess the correlation of TFEB expression and the pathological and clinical factors. Survival was analyzed by using the KaplanMeier analysis and Cox's proportional hazard regression model. The standard two-tailed independent samples $t$-test was performed to compare the differences in two groups. The significance level was defined as $P<0.05$. All experiments were repeated three times $(* P<0.05, * * P<0.01)$.

\section{Results Expression of TFEB was inhibited in CRC tissues}

To investigate the expression level of TFEB in CRC, we first analyzed its expression levels in The Cancer Genome Atlas (TCGA) database, and we found that TFEB mRNA was lower expressed in CRC tissues than normal tissues (Figure S1A, $P<0.01)$. To further define the expression level of TFEB, real-time PCR and Western blot were used to detect TFEB mRNA and protein expression levels in five pairs of fresh specimens of CRC tissues and adjacent normal tissues. The results showed that both TFEB mRNA level (Figure 1A) and protein level (Figure 1B) were lower in CRC tissues than adjacent normal tissues. Then IHC was also used to evaluate TFEB expression in cancer and adjacent normal tissues in 120 patients with $\mathrm{CRC}$, and as expected, the expression levels of TFEB were also lower in CRC tissues than adjacent normal tissues $(P<0.01)$ (Figure $1 \mathrm{C}$ and D).

\section{High levels of TFEB expression are associated with aggressive clinical features}

We had known that the expression of TFEB was inhibited in CRC tissues. To further explore the role of TFEB in CRC progression, we intensively analyzed the correlations between TFEB expression level and each clinicopathological variables such as age, sex, differentiation grade, infiltration depth, metastasis status, and Dukes stage using the 


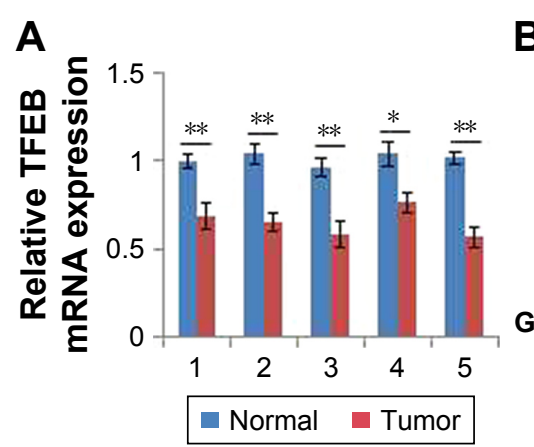

C

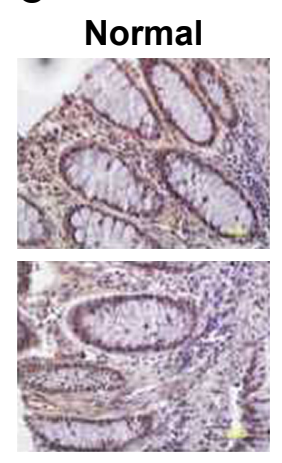

$\mathbf{F}$

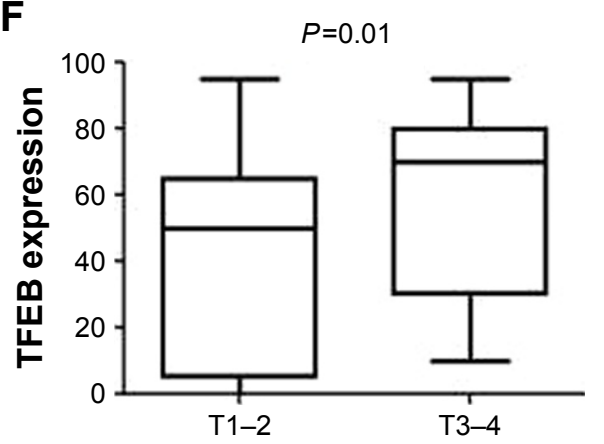

B

D
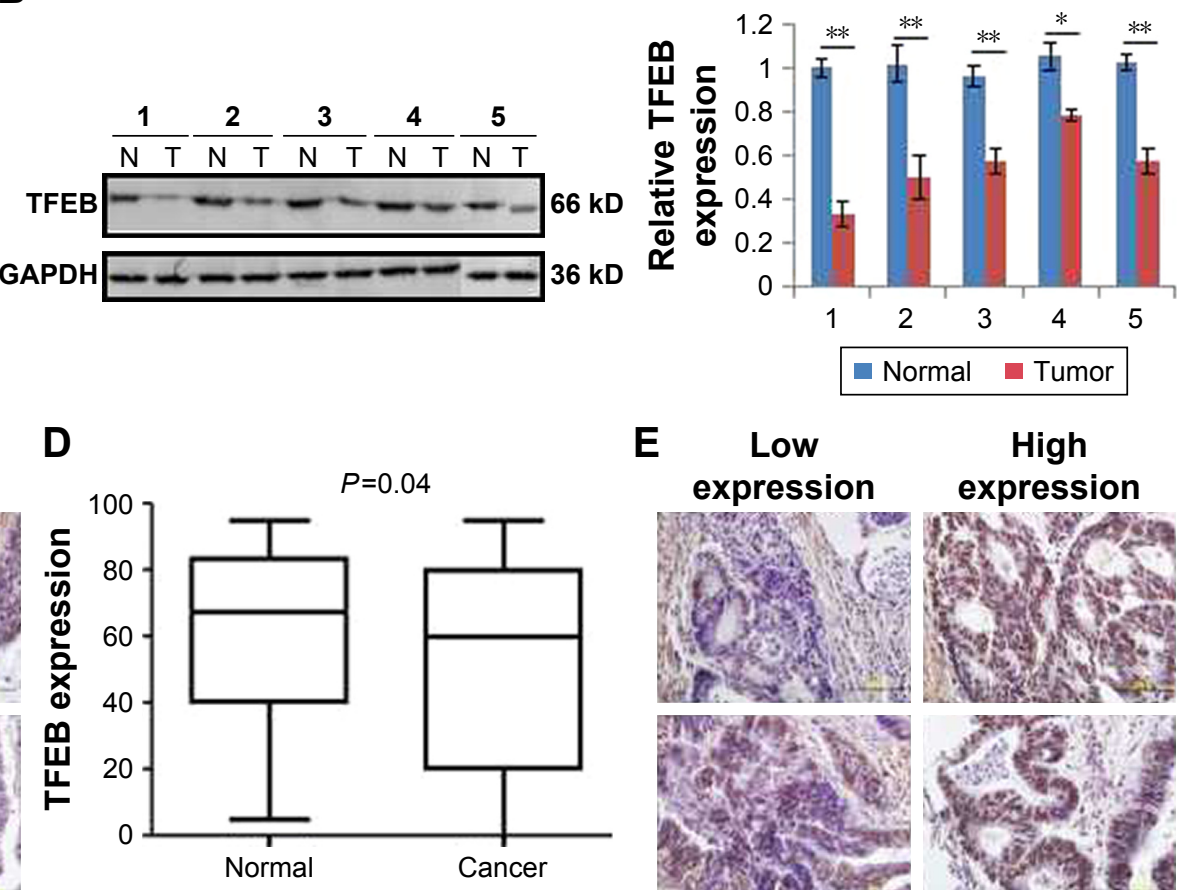

E

Low
expression

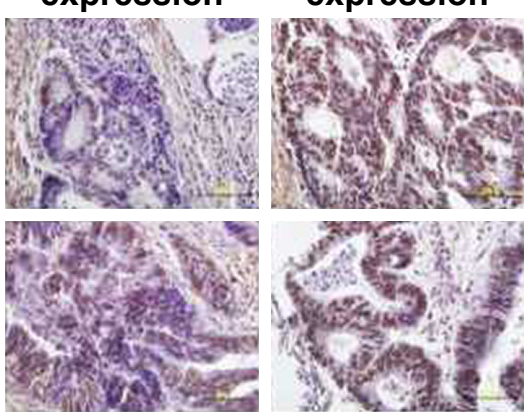

G

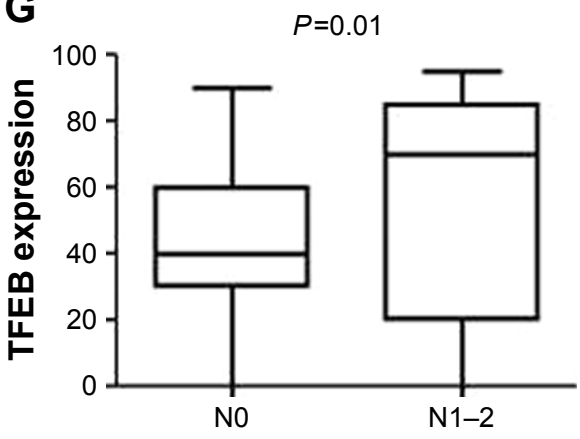

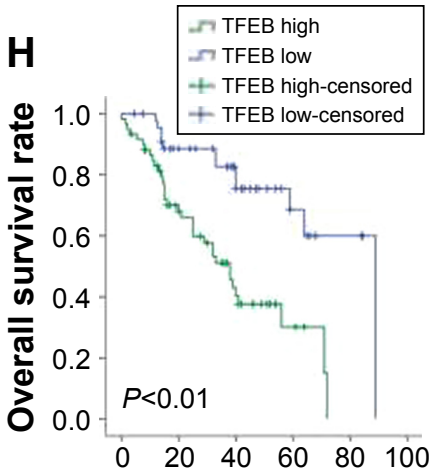

Survival time (month)

Figure I TFEB expression in CRC tissues and its association with the tumor progression.

Notes: (A and B) TFEB mRNA and protein expression in CRC and adjacent normal tissues. $* P<0.05$, $* * P<0.01$. (C and $\mathbf{D})$ IHC images and expression levels of TFEB in CRC tissues and adjacent normal tissues. (E) The present image of TFEB expression detected by IHC in CRC tissues. (F and $\mathbf{G})$ The correlations of TFEB expression level and infiltration depth, and TFEB and lymphatic metastasis. $(\mathbf{H})$ The significance of TFEB expression in the overall survival of the patients (IHC, 400 $)$.

Abbreviations: CRC, colorectal cancer; IHC, immunohistochemistry; TFEB, transcription factor EB.

TCGA database and our IHC results of TFEB. The results of TCGA database showed that there was no correlation between TFEB mRNA expression and age or sex (Figure S1B and C), but significant positive correlations between TFEB mRNA and infiltration depth, TFEB mRNA and lymphatic metastasis, TFEB mRNA and distant metastasis, and TFEB mRNA and clinical stage were found (Figure S1D-G). In addition, we analyzed the correlations of expression level of TFEB protein and clinicopathological variables in $120 \mathrm{CRC}$ cases. We divided these cases into two groups (low-expression group and high-expression group) according to TFEB IHC score (Figure 1E). The results are summarized in Table 1, which shows that there are positive correlations between TFEB and infiltration depth $(P=0.01$, Figure 1F) and TFEB and lymphatic metastasis $(P<0.01$, Figure 1G).

\section{Effect of TFEB on the prognosis of CRC patients}

We first analyzed the effect of TFEB mRNA on the prognosis of CRC patients using the database of TCGA, and we found that TFEB mRNA has no effect on the overall survival of 
Table I Correlations between TFEB expression and clinicopathological features

\begin{tabular}{|c|c|c|c|c|c|}
\hline \multirow[t]{2}{*}{ Variables } & \multirow[t]{2}{*}{$\mathbf{n}$} & \multicolumn{2}{|l|}{$\mathbf{N}$} & \multirow[t]{2}{*}{$\chi^{2}$} & \multirow[t]{2}{*}{$P$-value } \\
\hline & & $\begin{array}{l}\text { TFEB - } \\
\text { high }\end{array}$ & $\begin{array}{l}\text { TFEB - } \\
\text { low }\end{array}$ & & \\
\hline \multicolumn{6}{|l|}{ Age (years) } \\
\hline$<60$ & 71 & 42 & 29 & \multirow[t]{2}{*}{0.05} & \multirow[t]{2}{*}{0.83} \\
\hline$\geq 60$ & 49 & 28 & 21 & & \\
\hline \multicolumn{6}{|l|}{ Sex } \\
\hline Male & 81 & 43 & 38 & \multirow[t]{2}{*}{2.82} & \multirow[t]{2}{*}{0.09} \\
\hline Female & 39 & 27 & 12 & & \\
\hline \multicolumn{6}{|l|}{ Differentiation } \\
\hline Well-moderate & 76 & 44 & 32 & \multirow[t]{2}{*}{0.02} & \multirow[t]{2}{*}{0.90} \\
\hline Poor & 44 & 26 & 18 & & \\
\hline \multicolumn{6}{|l|}{ Infiltration depth } \\
\hline TI-T2 & 51 & 23 & 28 & \multirow[t]{2}{*}{6.39} & \multirow[t]{2}{*}{0.01} \\
\hline T3-T4 & 69 & 47 & 22 & & \\
\hline \multicolumn{6}{|c|}{ Lymphatic metastasis } \\
\hline No & 36 & 13 & 23 & \multirow[t]{2}{*}{10.45} & \multirow[t]{2}{*}{0.001} \\
\hline Yes & 84 & 57 & 27 & & \\
\hline \multicolumn{6}{|l|}{ Distant metastasis } \\
\hline No & 103 & 61 & 42 & \multirow[t]{2}{*}{0.24} & \multirow[t]{2}{*}{0.63} \\
\hline Yes & 17 & 9 & 8 & & \\
\hline \multicolumn{6}{|l|}{ Dukes stage } \\
\hline$A-B$ & 72 & 44 & 28 & \multirow[t]{2}{*}{0.57} & \multirow[t]{2}{*}{0.45} \\
\hline$C-D$ & 48 & 26 & 22 & & \\
\hline
\end{tabular}

Note: Bold values indicate $P<0.05$.

Abbreviation: TFEB, transcription factor EB.

CRC patients $(P=0.76$, Figure S1I), but progression-free survival rate was significantly lower in the TFEB mRNA higher-expression group $(P<0.01$, Figure $\mathrm{S} 1 \mathrm{H})$. Meanwhile, Kaplan-Meier survival analysis was used to analyze the effect of TFEB on the survival of patients in our 120 cases, and the results showed that the patients with higher TFEB expression always have poor survival $(P<0.01$, Figure $1 \mathrm{H})$. Furthermore, TFEB was a significant predictor of survival in multivariate analysis $(\mathrm{HR}=3.27 ; 95 \% \mathrm{CI}=1.13-7.58$, $P<0.01)$, when entered into a model consisting all clinicopathologic variables (Table 2).

\section{Silencing TFEB could inhibit cell growth and migration ability}

Since TFEB expression was correlated with aggressive features of CRC, to further explore the role of TFEB in the ability of cell growth and migration in vitro, we first knocked down TFEB in CRC cell lines of HCT116 and LoVo using shRNA (Figure 2A). Compared with shctrl cells, the growth and
Table 2 Multivariate analysis of survival in all population

\begin{tabular}{|l|l|l|l|l|}
\hline \multirow{2}{*}{ Variables } & HR & \multicolumn{2}{|l|}{ 95\% CI for HR } & \multirow{2}{*}{-value } \\
\cline { 3 - 5 } & & Lower & Upper & \\
\hline Sex (male vs female) & $0.7 \mathrm{I}$ & 0.34 & $\mathrm{I} .48$ & 0.35 \\
\hline Age, years (<60 vs $\geq 60$ ) & $\mathrm{I} .45$ & 0.72 & 2.93 & 0.30 \\
\hline Differentiation (well vs poor) & $\mathrm{I} .84$ & 0.85 & 3.96 & 0.12 \\
\hline $\begin{array}{l}\text { Infiltration depth (TI, T2 vs T3, } \\
\text { T4) }\end{array}$ & 0.85 & 0.32 & 2.29 & 0.75 \\
\hline Lymphatic metastasis (no vs yes) & 0.97 & $0.3 \mathrm{I}$ & 3.05 & 0.96 \\
\hline Distant metastases (no vs yes) & 0.89 & $0.3 \mathrm{I}$ & 2.57 & 0.83 \\
\hline Dukes stage (I/II vs III/IV) & 4.23 & 2.13 & $8.4 \mathrm{I}$ & $<\mathbf{0 . 0 I}$ \\
\hline TFEB (low vs high) & 3.27 & 1.13 & 7.58 & $<\mathbf{0 . 0 I}$ \\
\hline
\end{tabular}

Note: Bold values indicate $P<0.05$.

Abbreviation: TFEB, transcription factor EB.

colony formation ability of shTFEB cells were not affected in both HCT116 and LoVo (Figure 2B and C). Meanwhile, we also detected the change of migration ability of shTFEB cells and shctrl, but no difference was found in normal medium (Figure 2F). In consideration that TFEB is associated with autophagy, we thought whether shTFEB could affect the cell biology function when autophagy was induced, then the experiments were performed in AA-free medium. The results showed that shTFEB could significantly affect cell proliferation (Figure 2D), colony formation (Figure 2E), and migration ability (Figure 2G) in both HCT116 and LoVo in AA-free medium, which indicated that the role of TFEB in cell functions was more prominent in the cell medium of nutrient insufficiency.

Furthermore, we also constructed the cell line of sgTFEB in HCT116 using lentiCRISPR/CAS9 system, and the expression level of TFEB was significantly silenced in both protein level (Figure 3A) and mRNA level (Figure 3B). Similar experiments were also performed to detect the change of cell growth and migration ability in SgTFEB and sgctrl cells in AA-free medium, and the results showed that cell proliferation (Figure 3C), colony formation (Figure 3D), and migration ability (Figure 3E) were all inhibited in sgTFEB cells. When TFEB was resumed in sgTFEB, the abilities of cell proliferation and migration were resumed (Figure 3). Meanwhile, the animal experiment was also performed. We found that the tumor volumes of sgTFEB cells were significantly smaller than sgctrl cells, and TFEB overexpression could rescue the tumor growth (Figure 3F). Furthermore, the positive cells of Ki-67 were significantly decreased and the positive cells of Caspase 3 were significantly increased in sgTFEB tumors (Figure $3 \mathrm{G}$ and $\mathrm{H}$ ). All the results suggested that silencing TFEB could significantly inhibit cell proliferation 
A

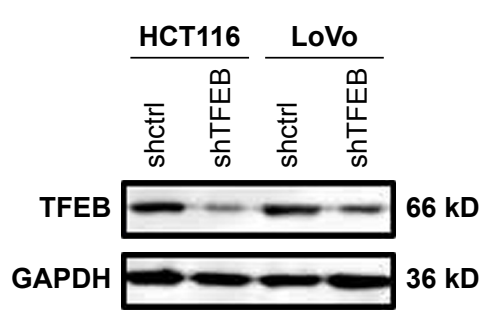

C
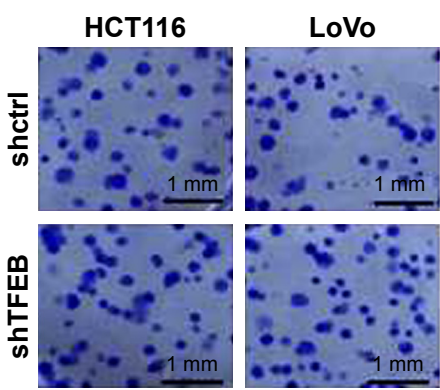

E
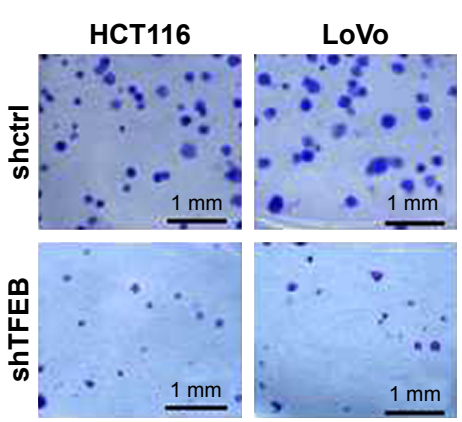

B

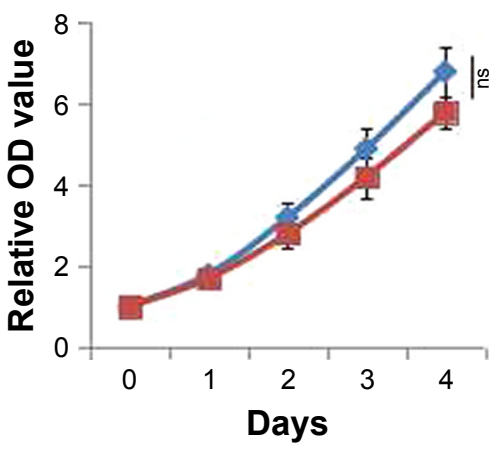

LoVo

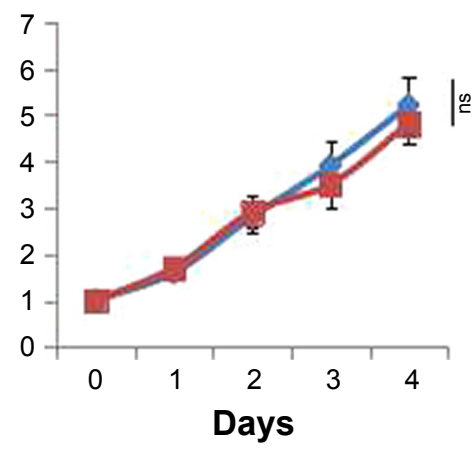

$\Rightarrow$ shctrl $-\mathbb{E}=$ shTFEB
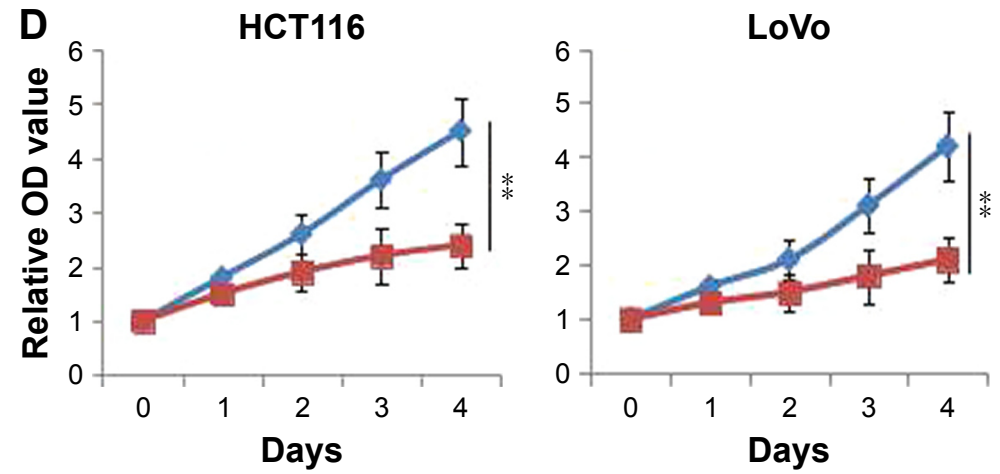

$\longrightarrow$ shctrl -2 - shTFEB
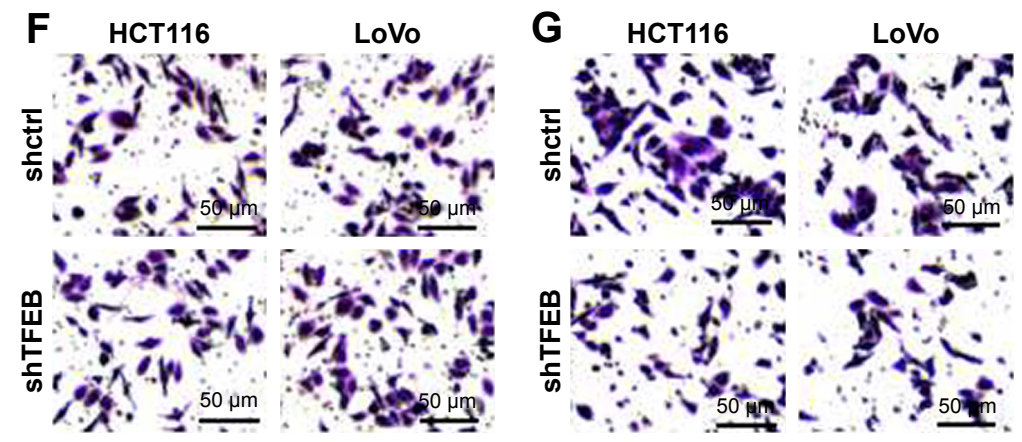

Figure 2 The effects of TFEB knockdown on cell ability of proliferation and migration.

Notes: (A) TFEB knockdown was established by shTFEB. (B) CCK-8 was used to detect cell proliferation in full medium. (C) Images of two-dimensional colony of shctrl and shTFEB cells in full medium. (D) CCK-8 was used to detect cell proliferation in AA-free medium. **P<0.0I. (E) Images of two-dimensional colony in AA-free medium. (F) Images of transwell in full medium. (G) Images of transwell in AA-free medium.

Abbreviations: CCK-8, Cell Counting Kit-8; ns, no significant difference; TFEB, transcription factor EB.

and migration ability in CRC cells in nutrient insufficiency microenvironment.

\section{Silencing TFEB inhibited the expression of Beclin I in CRC cells}

Since we found that silencing TFEB could significantly inhibit cell biology function in CRC cells in nutrient insufficiency microenvironment, we indicated that TFEB may play an important role in autophagy in CRC. Many reports have shown that TFEB could induce the expression of autophagy-associated proteins, including Beclin1. We first analyzed the correlation of TFEB and Beclin1 mRNA expression using TCGA database and found a positive correlation between these two genes (Figure 4A). Meanwhile, we also analyzed the correlation of TFEB and Beclin1 protein expression using the data of our IHC score (Figure 4B), and a significantly positive correlation was also found (Figure 4C). In addition, the protein level of 

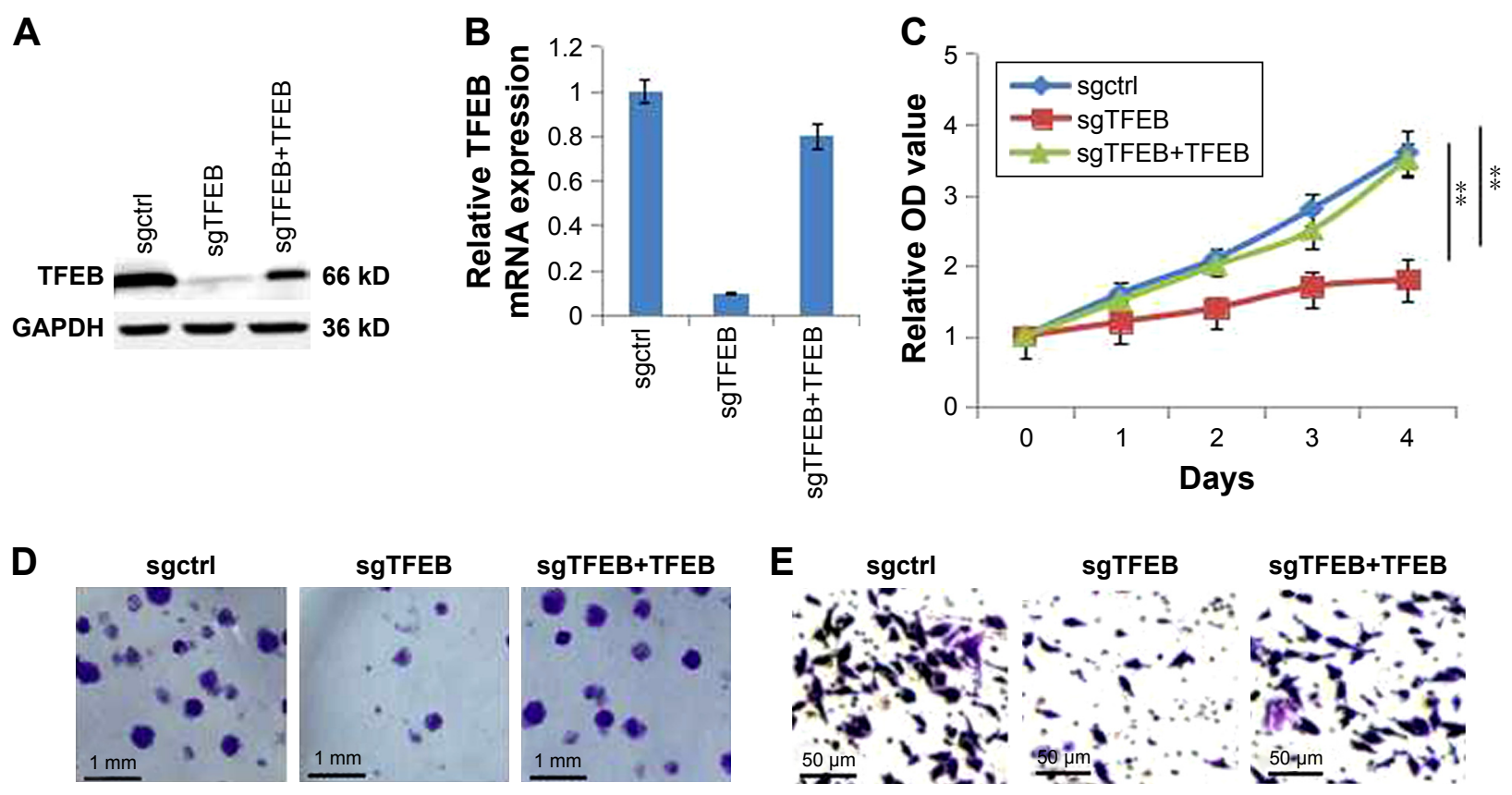

$\mathbf{F}$
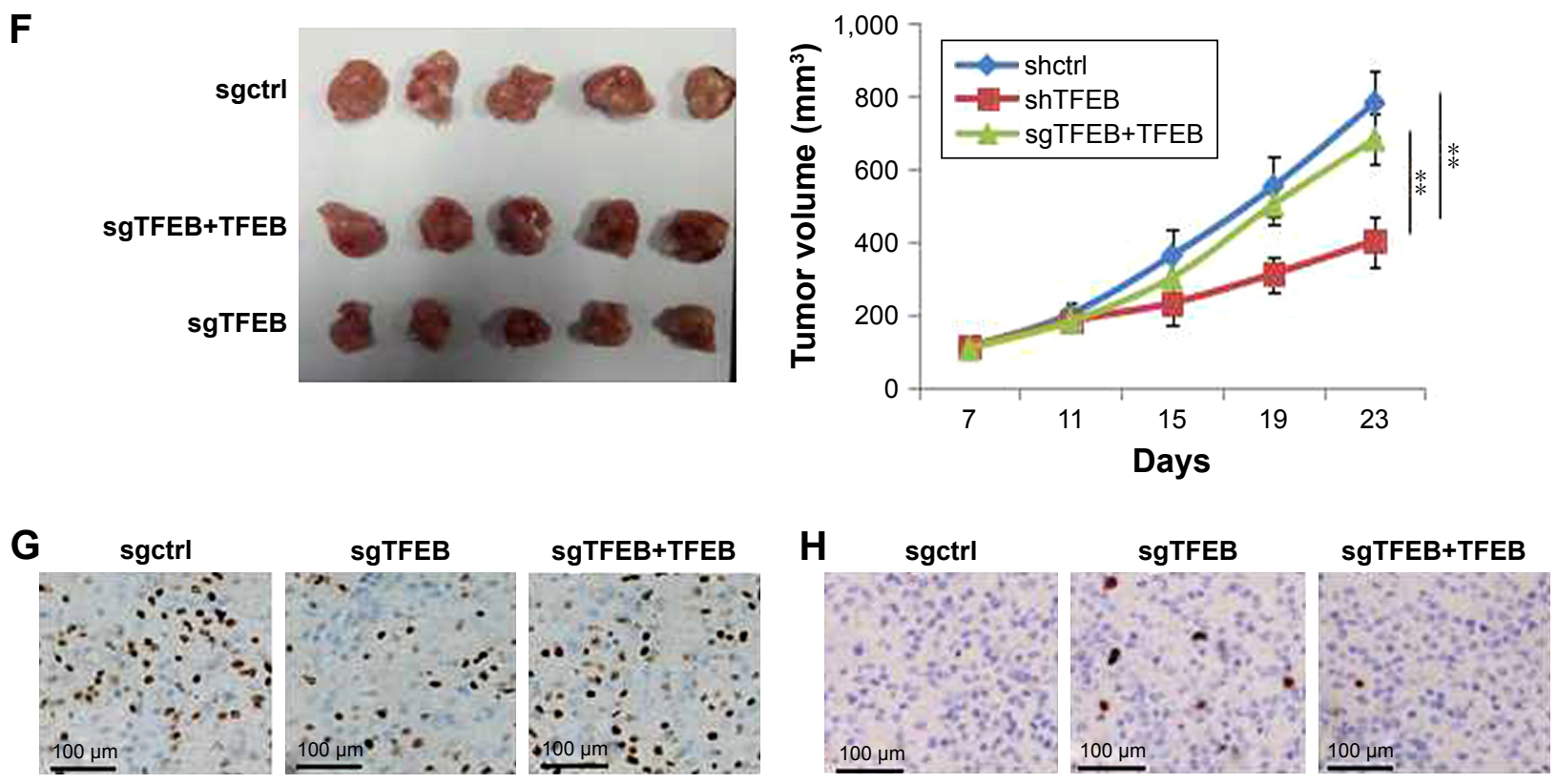

Figure 3 TFEB knockout significantly affect cell ability of proliferation and migration in AA-free medium.

Notes: (A) TFEB knockout was established by sgTFEB and rescued by TFEB overexpression in HCTII6. (B) The level of TFEB mRNA in sgctrl, sgTFEB, and sgTFEB-TFEB cells. (C) CCK-8 was used to detect these cell proliferation in AA-free medium. (D) Images of two-dimensional colony in AA-free medium. (E) Images of transwell in AA-free medium. (F) The effect of TFEB on tumor growth in vivo animal experiment. ( $\mathbf{G}$ and $\mathbf{H})$ The expression of Ki-67 and caspase-3 in tumor. ${ }^{* * P}<0.01$.

Abbreviations: CCK-8, Cell Counting Kit-8; TFEB, transcription factor EB.

Beclin1 was also significantly decreased in shTFEB cells in both HCT116 and LoVo (Figure 4D). As a transcription factor, luciferase reporter assay was used to detect the effect of TFEB on Beclin1 expression and showed that depletion of TFEB powerfully suppressed Beclin1 promoter ability (Figure 4E), and silencing TFEB could significantly inhibit Beclin1 expression in transcription level (Figure 4F).

\section{Discussion}

From the results, we found that, compared with adjacent normal tissues, the expression of TFEB was inhibited in CRC tissues. However, TFEB higher expression in CRC tissues was correlated with malignant progression of CRC and poor survival of CRC patients. In addition, silencing TFEB could significantly inhibit CRC cell ability of proliferation 

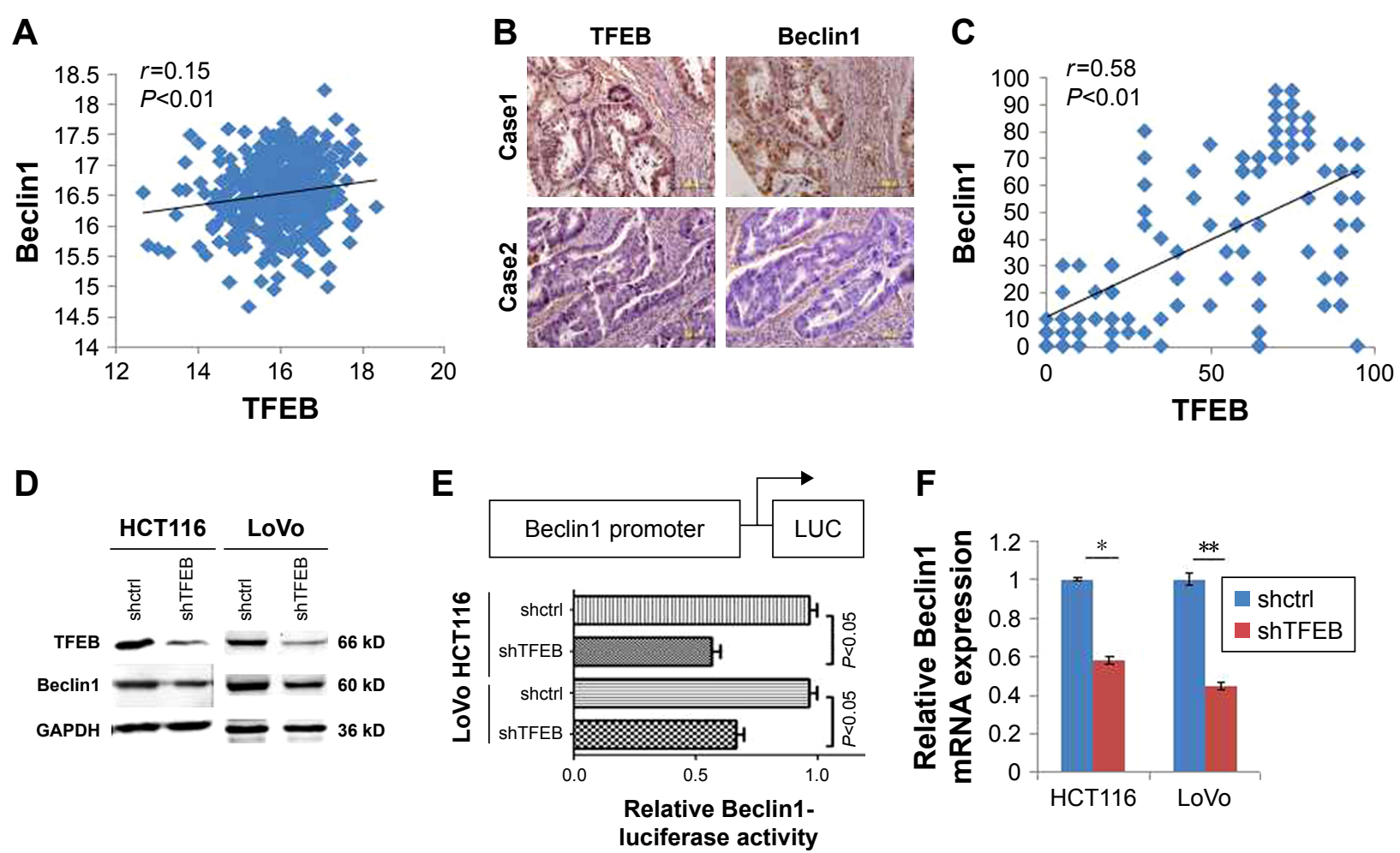

Figure 4 TFEB affects the expression of Beclin l.

Notes: (A) The correlation of TFEB and Beclin I mRNA in TCGA database. (B and C) IHC pictures and the correlation of TFEB and Beclin I expression (IHC 400x). (D) The effect of TFEB on Beclin I protein expression in CRC cell lines. (E) The effect of TFEB on Beclin I promoter activity. (F) The effect of TFEB on Beclin I mRNA expression. $* P<0.05$, $* * P<0.01$.

Abbreviations: IHC, immunohistochemistry; TFEB, transcription factor EB.

and migration in AA-free medium in vitro and inhibit tumor proliferation in vivo. All these results powerfully suggested that the higher expression of TFEB could accelerate cell proliferation and migration, which demonstrated that TFEB pathway may be a prognostic indicator and therapeutic target for CRC.

TFEB, a master transcription factor, could directly bind to the promoters of several autophagic lysosomal genes and lysosomal enzymes to promote their expression and regulate the autophagy progression in many kinds of cells, ${ }^{13}$ whereas the depletion of TFEB could inhibit the expression of lysosomal genes and affect intracellular clearance system in multiple cell types. ${ }^{20,21}$ In addition, TFEB also plays an important role in modulating organelle-specific autophagy through enhancing degradation of bulk autophagy substrates. ${ }^{22}$ Beclin1, interacting with PI3K, plays an important role in a critical step of the autophagic process. ${ }^{12}$ In our study, we found that there was a close correlation between TFEB and Beclin1 expression in CRC tissues, and silencing TFEB could significantly inhibit the promoter activity of Beclin1 and its expression in CRC cell lines.

Some papers have studied the oncogenic features of TFEB in cancer and found that chromosomal translocation of
TFEB could cause a particular type of renal cancer. ${ }^{23}$ In addition to chromosomal translocation inducing renal cancer, TFEB could also induce renal cancer through amplification. ${ }^{24}$ Meanwhile, TFEB overexpression could promote the growth of pancreatic adenoductal carcinoma via inducing autophagy, ${ }^{25}$ and TFEB was also identified as being highly expressed in non-Hodgkin's lymphoma. ${ }^{26}$ In this study, the expression of TFEB was inhibited in CRC tissues, however, consistent with other cancers, high levels of TFEB expression in CRC tissues are associated with aggressive clinical features and poor survival of CRC patients.

About autophagy in tumor genesis, the current hypothesis is that autophagy has dual and contradictory roles. In normal cells, autophagy could protect cells from malignant transformations. However, in tumor cells, autophagy could support tumor formation and metastasis in advanced stage. ${ }^{10}$ In our study, we think that it does not contradict that TFEB was highly expressed in normal colorectal tissues, in which TFEB and autophagy play a vital role. But when tumor has been formed or in late stage, TFEB would be highly expressed in $\mathrm{CRC}$ to regulate autophagy to promote tumor growth and metastasis. Hence, higher TFEB expression in CRC tissues was correlated with malignant progression of $\mathrm{CRC}$ and poor 
survival of CRC patients, and silencing TFEB in CRC cells could successfully inhibit cell growth and migration in nutrient deficiency microenvironment.

\section{Conclusion}

In conclusion, TFEB expression was inhibited in CRC cells, but higher TFEB expression correlated with aggressive clinical features in CRC. Meanwhile, higher TFEB expression was a prognostic factor of CRC. TFEB regulated cell autophagy through promoting Beclin1 expression; however, it is likely that future studies will have to address the identification of other proteins regulated by TFEB in CRC cells.

\section{Acknowledgments}

We appreciate all volunteers who participated in this study. And we also sincerely thank Tianjin Medical University Cancer Institute and Hospital for their assistance in experiments.

\section{Disclosure}

The authors report no conflicts of interest in this work.

\section{References}

1. Marley AR, Nan H. Epidemiology of colorectal cancer. Int $J$ Mol Epidemiol Genet. 2016;7(3):105-114.

2. Brody H. Colorectal cancer. Nature. 2015;521(7551):S1.

3. Hines RB, Jiban MJH, Choudhury K, et al. Post-treatment surveillance testing of patients with colorectal cancer and the association with survival: protocol for a retrospective cohort study of the Surveillance, Epidemiology, and End Results (SEER)-medicare database. BMJ Open. 2018;8(4):e022393.

4. Holch JW, Demmer M, Lamersdorf C, et al. Pattern and dynamics of distant metastases in metastatic colorectal cancer. Visc Med. 2017; 33(1):70-75.

5. Samawi HH, Shaheen AA, Tang PA, Heng DYC, Cheung WY, Vickers MM. Risk and predictors of suicide in colorectal cancer patients: a Surveillance, Epidemiology, and End Results analysis. Curr Oncol. 2017;24(6):e513-e517.

6. Zarkavelis G, Boussios S, Papadaki A, Katsanos KH, Christodoulou DK, Pentheroudakis G. Current and future biomarkers in colorectal cancer. Ann Gastroenterol. 2017;30(6):613-621.

7. Cuyle PJ, Prenen H. Current and future biomarkers in the treatment of colorectal cancer. Acta Clin Belg. 2017;72(2):103-115.

8. Yu T, Guo F, Yu Y, et al. Fusobacterium nucleatum promotes chemoresistance to colorectal cancer by modulating autophagy. Cell. 2017; 170(3):e516:548-563.
9. $\mathrm{Hu} \mathrm{JL}, \mathrm{He}$ GY, Lan XL, et al. Inhibition of ATG12-mediated autophagy by miR-214 enhances radiosensitivity in colorectal cancer. Oncogenesis. 2018;7(2):16.

10. Wilde L, Tanson K, Curry J, Martinez-Outschoorn U. Autophagy in cancer: a complex relationship. Biochem J. 2018;475(11):1939-1954.

11. Russo M, Russo GL. Autophagy inducers in cancer. Biochem Pharmacol. 2018;153:51-61.

12. Zhang J, Wang G, Zhou Y, Chen Y, Ouyang L, Liu B. Mechanisms of autophagy and relevant small-molecule compounds for targeted cancer therapy. Cell Mol Life Sci. 2018;75(10):1803-1826.

13. Napolitano G, Ballabio A. TFEB at a glance. J Cell Sci. 2016;129(13): 2475-2481.

14. Nezich CL, Wang C, Fogel AI, Youle RJ. MiT/TFE transcription factors are activated during mitophagy downstream of Parkin and Atg5. J Cell Biol. 2015;210(3):435-450.

15. Li L, Sun B, Gao Y, Niu H, Yuan H, Lou H. STAT3 contributes to lysosomal-mediated cell death in a novel derivative of riccardin D-treated breast cancer cells in association with TFEB. Biochem Pharmacol. 2018;150:267-279.

16. Klein K, Werner K, Teske C, et al. Role of TFEB-driven autophagy regulation in pancreatic cancer treatment. Int J Oncol. 2016;49(1): 164-172.

17. Giatromanolaki A, Kalamida D, Sivridis E, et al. Increased expression of transcription factor EB (TFEB) is associated with autophagy, migratory phenotype and poor prognosis in non-small cell lung cancer. Lung Cancer. 2015;90(1):98-105.

18. Zeng C, Matsuda K, Jia WH, et al. Identification of susceptibility loci and genes for colorectal cancer risk. Gastroenterology. 2016;150(7): $1633-1645$.

19. Joung J, Konermann S, Gootenberg JS, et al. Genome-scale CRISPRCas9 knockout and transcriptional activation screening. Nat Protoc. 2017;12(4):828-863.

20. Ploper D, Taelman VF, Robert L, et al. MITF drives endolysosomal biogenesis and potentiates Wnt signaling in melanoma cells. Proc Natl Acad Sci U S A. 2015;112(5):E420-E429.

21. Medina DL, Fraldi A, Bouche V, et al. Transcriptional activation of lysosomal exocytosis promotes cellular clearance. Dev Cell. 2011;21(3): 421-430.

22. Settembre C, de Cegli R, Mansueto G, et al. TFEB controls cellular lipid metabolism through a starvation-induced autoregulatory loop. Nat Cell Biol. 2013;15(6):647-658.

23. Malouf GG, Su X, Yao H, et al. Next-generation sequencing of translocation renal cell carcinoma reveals novel RNA splicing partners and frequent mutations of chromatin-remodeling genes. Clin Cancer Res. 2014;20(15):4129-4140.

24. Durinck S, Stawiski EW, Pavía-Jiménez A, et al. Spectrum of diverse genomic alterations define non-clear cell renal carcinoma subtypes. Nat Genet. 2015;47(1):13-21.

25. Perera RM, Stoykova S, Nicolay BN, et al. Transcriptional control of autophagy-lysosome function drives pancreatic cancer metabolism. Nature. 2015;524(7565):361-365.

26. Gayle S, Landrette S, Beeharry N, et al. Identification of apilimod as a first-in-class PIKfyve kinase inhibitor for treatment of B-cell nonHodgkin lymphoma. Blood. 2017;129(13):1768-1778. 


\section{Supplementary material}
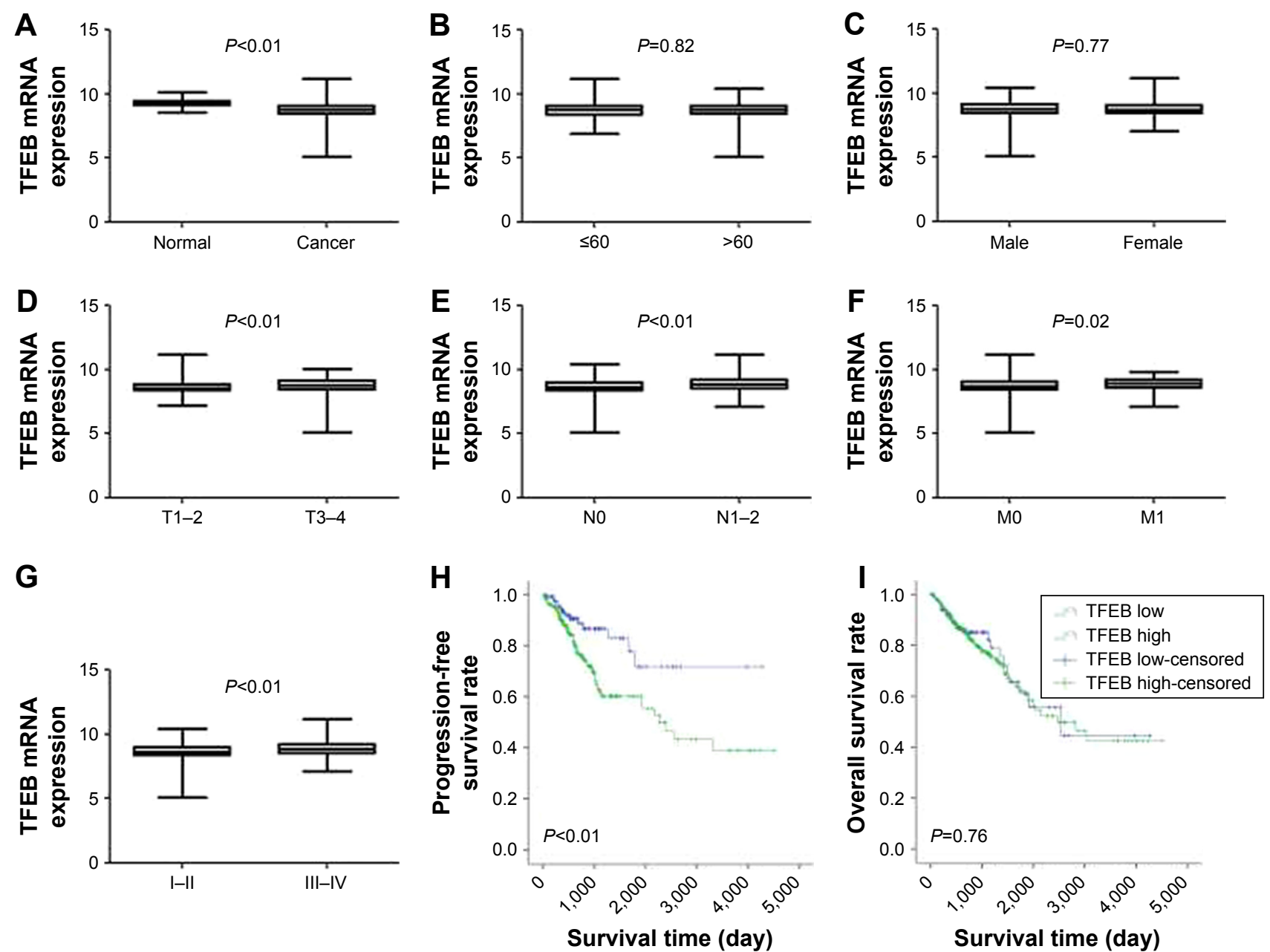

Figure SI TFEB expression in CRC in TCGA database.

Notes: (A) TFEB mRNA expression in CRC and normal tissues. (B-G) The correlations of TFEB mRNA expression level and age, sex, infiltration depth, lymphatic metastasis, or distant metastasis. (H) The significance of TFEB mRNA in progression-free survival. (I) The significance of TFEB mRNA in overall survival. Abbreviations: CRC, colorectal cancer; TFEB, transcription factor EB.

\section{Publish your work in this journal}

OncoTargets and Therapy is an international, peer-reviewed, open access journal focusing on the pathological basis of all cancers, potential targets for therapy and treatment protocols employed to improve the management of cancer patients. The journal also focuses on the impact of management programs and new therapeutic agents and protocols on patient perspectives such as quality of life, adherence and satisfaction. The manuscript management system is completely online and includes a very quick and fair peer-review system, which is all easy to use. Visit http://www.dovepress.com/testimonials.php to read real quotes from published authors. 